\title{
CD73 or CD39 Deletion Reveals Different Mechanisms of Formation for Spontaneous and Mechanically-Stimulated Adenosine and Sex Specific Compensations in ATP Degradation
}

\author{
Ying Wang, ${ }^{1}$ Jeffrey Copeland,,${ }^{1,2}$ Mimi Shin,${ }^{1}$ Yuanyu Chang, ${ }^{1}$ B. Jill Venton ${ }^{1}$ \\ ${ }^{1}$ Dept. of Chemistry, University of Virginia, Charlottesville, VA 22902 \\ 2Department of Biology, Eastern Mennonite University, Harrisonburg, VA 22802
}




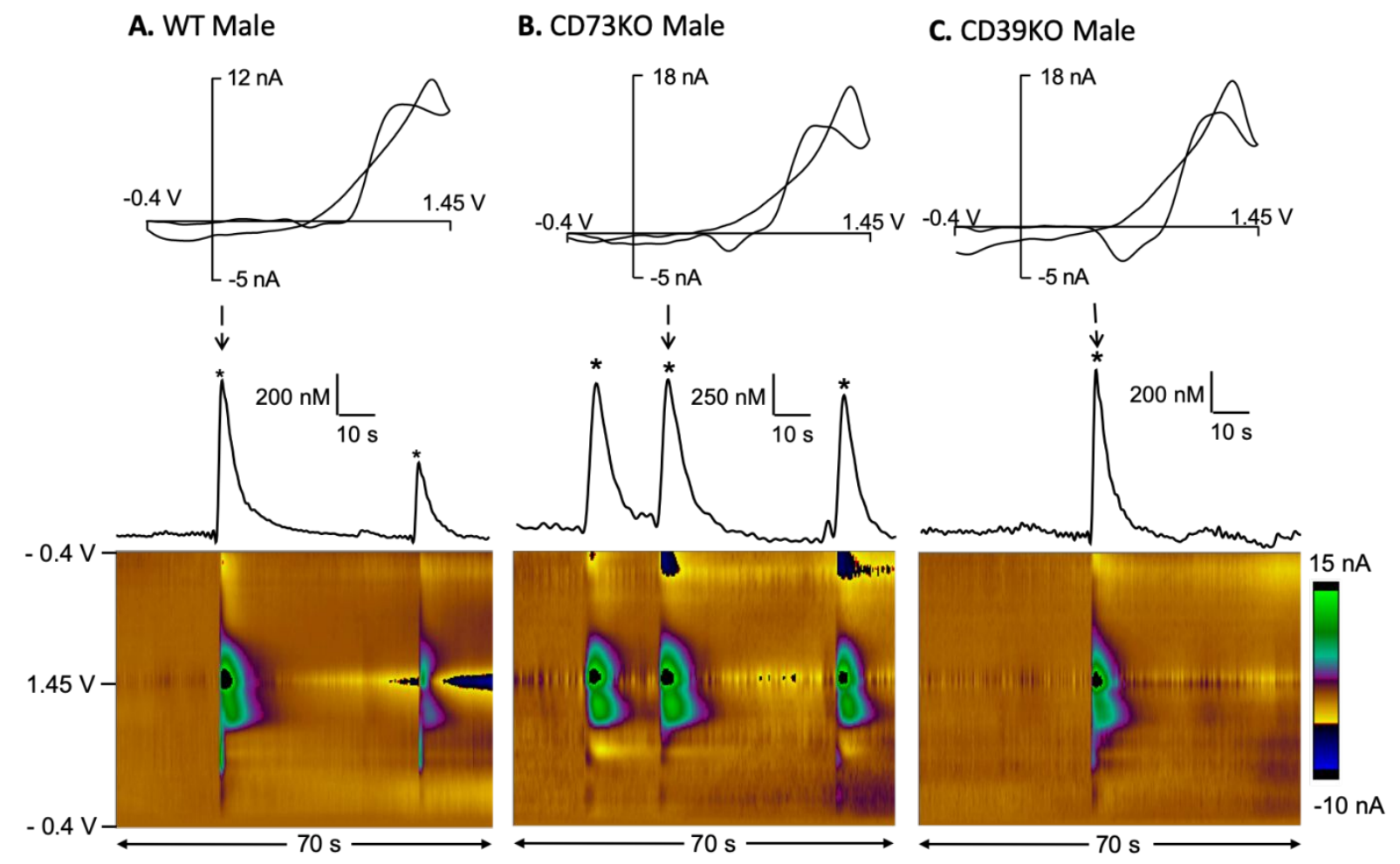

Figure S1. Spontaneous adenosine in the hippocampus. Example adenosine events in the males of (A) wild-type, (B) CD73KO, and (C) CD39KO mice. Adenosine can be identified in the cyclic voltammograms (top) by its primary oxidation peak at $1.3 \mathrm{~V}$ on the cathodic scan and the secondary oxidation peak at $1.2 \mathrm{~V}$ on the anodic scan. Concentration vs. time traces (middle) were derived from the corresponding 3-D color plots (bottom). Adenosine oxidations are the green/purple area in the middle of the color plot. 

A. Wild-type Male
B. CD73KO Male
C. CD39KO Male

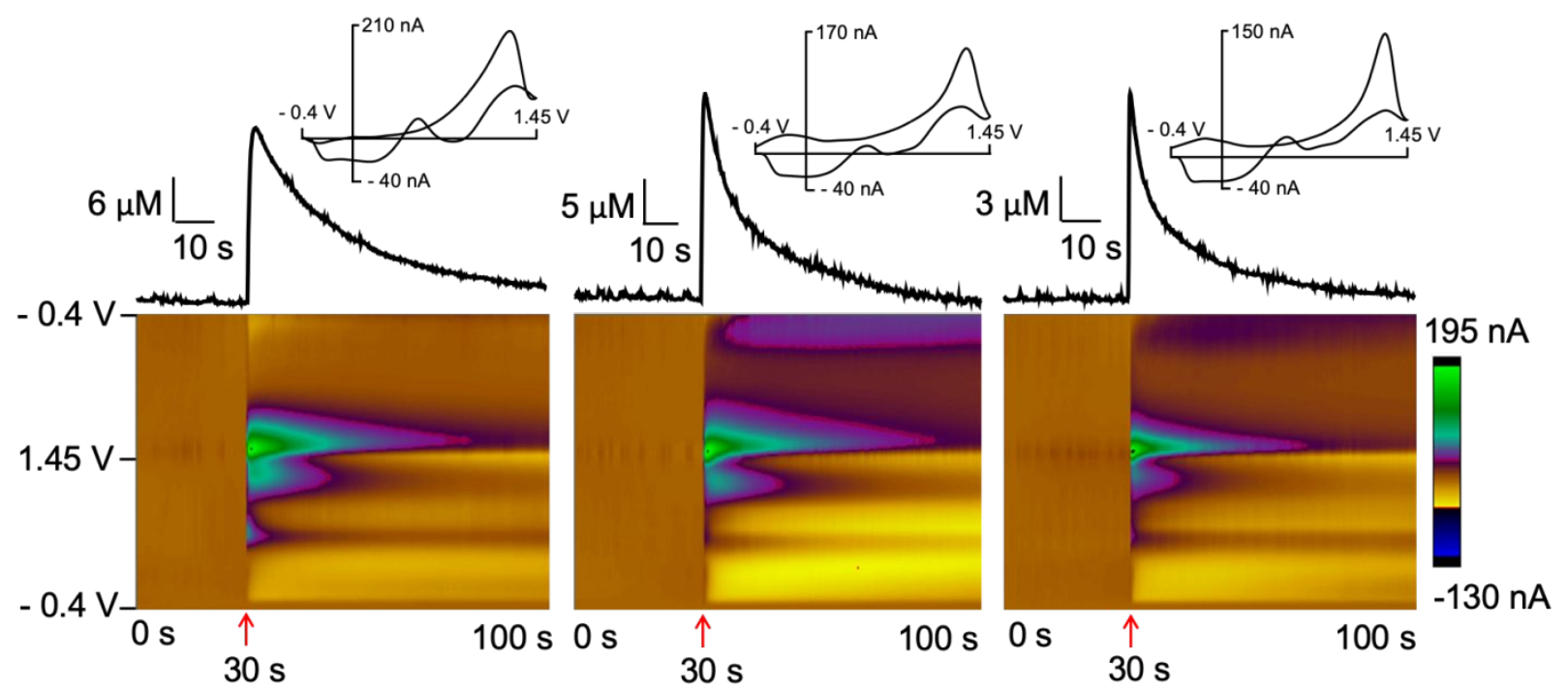

Figure S2. Mechanically-stimulated adenosine in the hippocampus. Example of mechanically-stimulated adenosine in male (A) wild-type, (B) CD73KO, and (C) CD39KO mice. Electrode was lowered $0.1 \mathrm{~mm}$ at $30 \mathrm{~s}$ (arrow). Concentration vs. time traces show the peak concentration of adenosine. Four consecutive stimulations were performed every 15 min for totally 4 stimulations. 
Table S1. Full 2-way ANOVA post-test stats for Fig. 3A: Number of Spontaneous Adenosine Transient Events

\begin{tabular}{|c|c|c|c|}
\hline Group 1 & Group 2 & P value & Significance \\
\hline WT Male & CD73KO Male & 0.9689 & ns \\
\hline WT Male & CD39KO Male & 0.0499 & * \\
\hline WT Male & WT Female & 0.5947 & ns \\
\hline WT Male & CD73KO Female & 0.0911 & ns \\
\hline WT Male & CD39KO Female & 0.1911 & ns \\
\hline CD73KO Male & CD39KO Male & 0.0102 & * \\
\hline CD73KO Male & WT Female & 0.9532 & ns \\
\hline CD73KO Male & CD73KO Female & 0.0196 & * \\
\hline CD73KO Male & CD39KO Female & 0.0458 & * \\
\hline CD39KO Male & WT Female & 0.0017 & ** \\
\hline CD39KO Male & CD73KO Female & 0.9995 & ns \\
\hline CD39KO Male & CD39KO Female & 0.9756 & ns \\
\hline WT Female & CD73KO Female & 0.0033 & ** \\
\hline WT Female & CD39KO Female & 0.0079 & ** \\
\hline CD73KO Female & CD39KO Female & 0.9981 & ns \\
\hline
\end{tabular}

ns $=$ not significant ${ }^{*} p<0.05^{* *} p<0.01,{ }^{* * *} p<0.001,{ }^{* * * *} p<0.0001$

Table S2. Full 2-way ANOVA post-test stats for Fig. 3B, Average Concentration of Spontaneous Adenosine Events

\begin{tabular}{|c|c|c|c|}
\hline Group 1 & Group 2 & P value & Significance \\
\hline WT Male & CD73KO Male & 0.0157 & * \\
\hline WT Male & CD39KO Male & 0.9910 & ns \\
\hline WT Male & WT Female & 0.0012 & $* *$ \\
\hline WT Male & CD73KO Female & 0.8597 & ns \\
\hline WT Male & CD39KO Female & $<0.0001$ & $* * * *$ \\
\hline CD73KO Male & CD39KO Male & 0.0018 & ** \\
\hline CD73KO Male & WT Female & 0.9824 & ns \\
\hline CD73KO Male & CD73KO Female & 0.0002 & $* * *$ \\
\hline CD73KO Male & CD39KO Female & 0.0972 & ns \\
\hline CD39KO Male & WT Female & $<0.0001$ & $* * * *$ \\
\hline CD39KO Male & CD73KO Female & 0.9940 & ns \\
\hline CD39KO Male & CD39KO Female & $<0.0001$ & $* * * *$ \\
\hline WT Female & CD73KO Female & $<0.0001$ & $\star \star \star \star *$ \\
\hline WT Female & CD39KO Female & 0.3984 & ns \\
\hline CD73KO Female & CD39KO Female & $<0.0001$ & $* * * *$ \\
\hline
\end{tabular}

ns=not significant ${ }^{*} p<0.05^{* *} p<0.01,{ }^{* * *} p<0.001,{ }^{* * * *} p<0.0001$ 
Table S3. Full 2-way ANOVA post-test results for Fig. 3C: Average Concentration of Mechanically-Stimulated Adenosine

\begin{tabular}{|l|l|l|c|}
\hline Group 1 & Group 2 & P value & Significance \\
\hline WT Male & CD73KO Male & 0.9176 & $\mathrm{~ns}$ \\
\hline WT Male & CD39KO Male & 0.1061 & ns \\
\hline WT Male & WT Female & 0.0367 & ${ }^{* *}$ \\
\hline WT Male & CD73KO Female & 0.0089 & $\mathrm{~ns}$ \\
\hline WT Male & CD39KO Female & 0.2773 & $\mathrm{~ns}$ \\
\hline CD73KO Male & CD39KO Male & 0.4886 & $\mathrm{~ns}$ \\
\hline CD73KO Male & WT Female & 0.2256 & $\mathrm{~ns}$ \\
\hline CD73KO Male & CD73KO Female & 0.0663 & $\mathrm{~ns}$ \\
\hline CD73KO Male & CD39KO Female & 0.8143 & $\mathrm{~ns}$ \\
\hline CD39KO Male & WT Female & 0.9932 & $\mathrm{~ns}$ \\
\hline CD39KO Male & CD73KO Female & 0.8159 & $\mathrm{~ns}$ \\
\hline CD39KO Male & CD39KO Female & 0.9920 & $\mathrm{~ns}$ \\
\hline WT Female & CD73KO Female & 0.9815 & $\mathrm{~ns}$ \\
\hline WT Female & CD39KO Female & 0.8691 & \\
\hline CD73KO Female & CD39KO Female & 0.4905 & \\
\hline
\end{tabular}

ns=not significant, ${ }^{*} p<0.05^{* *} p<0.01,{ }^{* * *} p<0.001,{ }^{* * * *} p<0.0001$ 